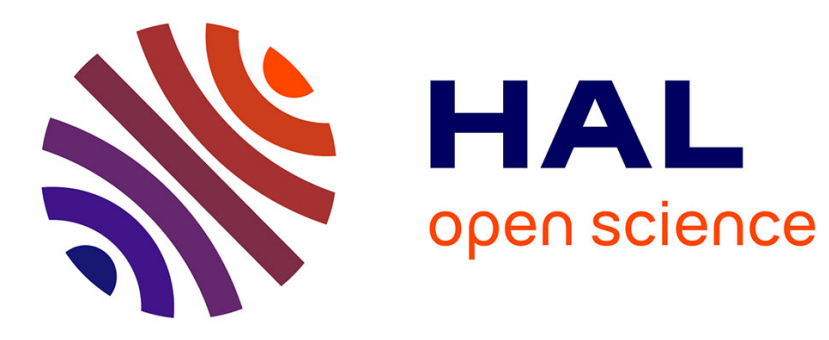

\title{
The Role of Ligaments: Patient-Specific or Scenario-Specific?
}

Julien Bosman, Nazim Haouchine, Jérémie Dequidt, Igor Peterlik, Stéphane Cotin, Christian Duriez

\section{- To cite this version:}

Julien Bosman, Nazim Haouchine, Jérémie Dequidt, Igor Peterlik, Stéphane Cotin, et al.. The Role of Ligaments: Patient-Specific or Scenario-Specific?. International Symposium on Biomedical Simulation ISBMS, Oct 2014, Strasbourg, France. hal-01068077

\section{HAL Id: hal-01068077 https://hal.inria.fr/hal-01068077}

Submitted on 24 Sep 2014

HAL is a multi-disciplinary open access archive for the deposit and dissemination of scientific research documents, whether they are published or not. The documents may come from teaching and research institutions in France or abroad, or from public or private research centers.
L'archive ouverte pluridisciplinaire HAL, est destinée au dépôt et à la diffusion de documents scientifiques de niveau recherche, publiés ou non, émanant des établissements d'enseignement et de recherche français ou étrangers, des laboratoires publics ou privés. 


\title{
The Role of Ligaments: Patient-Specific or Scenario-Specific?
}

\author{
Julien Bosman ${ }^{1,2}$, Nazim Haouchine ${ }^{1,2}$, Jeremie Dequidt $^{1,2}$, Igor Peterlik ${ }^{3,4}$, \\ Stéphane $\operatorname{Cotin}^{1,2,3}$, Christian Duriez ${ }^{1,2}$ \\ ${ }^{1}$ Shacra Team, INRIA \\ ${ }^{2}$ Lille University, France \\ ${ }^{3}$ IHU Strasbourg, France \\ ${ }^{4}$ Institute of Computer Science, Masaryk University, Czech Republic
}

\begin{abstract}
In this paper, we present a preliminary study dealing with the importance of correct modeling of connective tissues such as ligaments in laparoscopic liver surgery simulation. We show that the model of these tissues has a significant impact on the overall results of the simulation. This is demonstrated numerically using two different scenarios from the laparoscopic liver surgery, both resulting in important deformation of the liver: insufflation of the abdominal cavity with gas (pneumoperitoneum) and manipulation with the liver lobe using a surgical instrument (grasping pincers). For each scenario, a series of simulations is performed with or without modeling the deformation of the ligaments (fixed constraints or biomechanical model with the parameter of the literature). The numerical comparison shows that modeling the ligament deformations can be at least as important as the correct selection of the patient-specific parameters, nevertheless this observation depends on the simulated scenario.
\end{abstract}

\section{Introduction}

In the context of patient-specific simulations there is a strong need of precise biomechanical models, which are capable to capture and predict the deformations of the organs targeted during given procedure. The ultimate goal is to use these models in the pre-operative context in order to improve procedure planning as well as in the per-operative context, where model-based real-time simulation is used as a tool for guidance directly in the operation room.

When high accuracy of the biomechanical models is required, the finite element method seems to be the best approach to capture the deformations of tissues. This method is employed to integrate viscoelastic or hyperelastic constitutive laws over the geometric domain of an organ [4]. Intensive work has been done on measurement and estimation of soft tissue constitutive laws [6,3], nevertheless, it is still unclear where the effort should be put to obtain accurate and realistic results [5]. We propose to investigate the role of the surrounding tissues and the boundary conditions that are often under-estimated. For instance, while the constitutive laws of the liver tissues have been well studied $[9,7]$, to 
our best knowledge, there are only few existing works focusing on the role of the boundary conditions and the anatomical structures surrounding the liver.

In this paper, we focus on the role of ligaments; more precisely, we show that the modeling of ligament deformation has a significant impact on the overall simulation results. We use two different scenarios, typically performed in the context of laparoscopic surgery, both resulting in important deformations of the liver: insufflation of the abdominal cavity with gas (pneumoperitoneum) and manipulation of the liver lobe using a surgical instrument (graspers). For each scenario, a series of simulations is performed using different methods for modeling of ligaments (fixed constraints and biomechanical model). We show that removing the ligament deformation has a significant influence on the simulation of the liver. We compare this to the influence of the constitutive law used to model the liver deformation, using numerical test based on under- or over-estimation of the material properties. Then we note that given the type of input loading used to deform the model (displacement or effort), the influence changes.

\section{Methods}

We perform the study in the context of laparoscopic liver surgery: the laparoscopic scene represents the right and left lobes separated on the anterior side by the falciform ligament (Fig. 1). The 3D model of the lobes is obtained from pre-operative CT-scans including the vascular network [7]. The falciform ligament is more difficult to extract from the pre-operative images, however, recent work [8] shows promising results in the transfer of the ligament positions using atlas-based techniques. Thus, we assume that the ligament position can be determined. The underlying fat supporting the liver is also modeled to simulate the surrounding connective tissues.

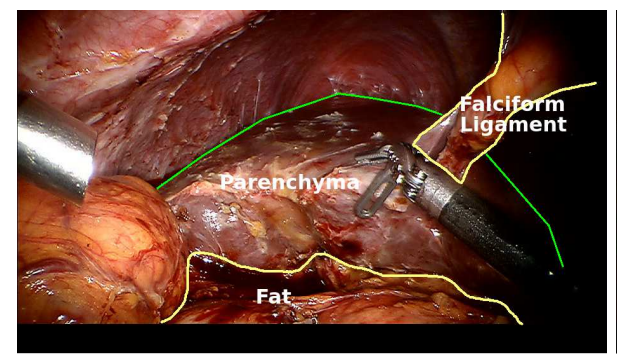

(a)

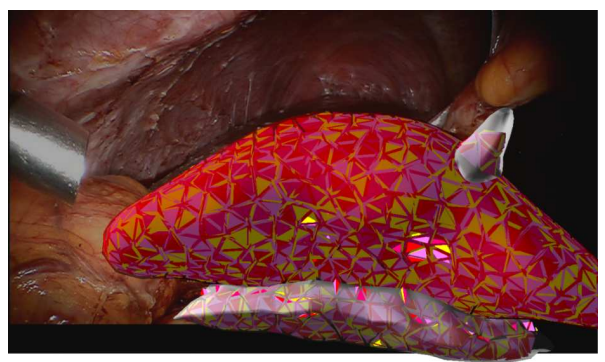

(b)

Fig. 1. Laparoscopic view of liver showing (a) contours of lobes, falciform ligament and surrounding fat, (b) superimposed FE meshes generated from pre-operative CT scans.

We perform three experiments: first, the liver is deformed with a surgical instrument with known position. Second, a force similar to that imposed by the 
instrument is applied to the surface of the liver. Third, liver deformation due to pneumoperitoneum is simulated given the pressure applied to the patient's abdomen. The important difference between these experiments is in the type of load that creates the deformation: in the first case, a displacement is imposed on a part of the mesh (in the region of the liver that is being grasped), whereas in the second and third cases a force and a pressure (an effort) is imposed on the surface of the model. In both cases, the goal is to obtain the displacement field of the nodes of the liver model.

As the aim of this work is to study the influence of boundary conditions, the simulation involves several tests in which we combine different methods to simulate the ligaments. In each test, the liver parenchyma is simulated with the corotational formulation of the finite element method employing the linear constitutive law.

As for the ligaments, two different simulations are performed, each being based on a different hypothesis. According to the first hypothesis, the ligament is considered a full anatomical structure and simulated using linear elasticity. Alternatively, the ligaments are considered stiff enough to be modeled as fixed points on the liver surface.

\section{Results}

The simulations are performed using SOFA framework[1]. The implicit integration based on backward Euler method is employed and the linearized system of equations is solved in each time step using the preconditioned conjugate gradients. The volume meshes are composed of linear tetrahedral elements.

In our reference simulation, the liver and the ligaments are simulated using finite element corotational formulation. In the three experiments, we compare the results of each test to the reference simulation. The error evaluation on the displacement is done after the equilibrium is achieved using root mean square error (RMSE).

The tests are organized as follows: first we compare the role of the ligaments which are modeled either as fixed points or using using the corotational formulation with Young's modulus of $E_{\text {lig }}=150 \mathrm{kPa}$. Then, in the second and third tests, ligaments are simulated with FEM, but we under- and over-estimate the Young's modulus of the parenchyma (initially set to $E_{r e f}=27 \mathrm{kPa}$ ) by the factor of two w.r.t. the reference simulation.

The grasping is simulated by prescribing a displacement on the lobe of the liver. The motion of the displaced nodes is extracted from a video of a laparoscopic surgery. The maximum displacement is $75 \mathrm{~mm}$. To ensure that both prescribed displacements/efforts tests result in a similar motion, the prescribed force value is computed from the prescribed displacements scenario. We measure the average elastic force of the displaced nodes of the liver after the equilibrium. From our experiment, a traction force of $15.42 \mathrm{~N}$ has to be applied on the nodes to be equivalent to the prescribed displacements. The direction of the force vector is chosen to be the same as the one of the displacement vector. Displacements 


\begin{tabular}{lccc}
\hline Experiments & Grasping & Traction & Pressure \\
\hline Fixed nodes & 2.75 & 7.19 & 3.53 \\
$E=0.5 \times E_{\text {ref }}$ & 1.12 & 7.76 & 8.40 \\
$E=2 \times E_{\text {ref }}$ & 1.77 & 4.85 & 3.93
\end{tabular}

Table 1. Evaluation of the RMSE (in $\mathrm{mm}$ ) for prescribed displacement (grasping) and prescribed forces (traction and pressure), compared to our reference simulation.

and efforts are prescribed on the sames nodes. The pneumoperitoneum is modeled by applying a pressure of $12 \mathrm{mmHg}$ on the liver surface, which is an average pressure used by surgeons during abdominal laparoscopic surgery [2].

The results presented in Tab. 1 show two specific scenarios: simulations where the deformation is induced by prescribing a displacement and those where applied forces are prescribed, each of them having their own requirements.

Considering prescribed displacements, our results show that a large difference in the elasticity parameter of the liver has a relatively small impact on the error when compared to the influence of the modeling of the ligaments. The influence of the model used for ligaments is more than twice as important as the elasticity parameter used for the parenchyma. The results demonstrate the fact that in such a scenario, the use of a physically-based deformable model is crucial.

As for the prescribed forces, the influence of variation in Young's modulus of the parenchyma is much more significant than in the case of prescribed displacements. The results emphasize the importance of using patient-specific data in such scenario. However, in this case, they also show that the influence of the model used for ligaments is comparable to the impact of the elasticity parameters.

Going further, our results also show that the difference between errors due to the ligament model and to the elasticity parameter is important when simulating the same action, in our case pulling on a liver lobe, using two different loading methods (prescribed displacement/prescribed forces).

\section{Conclusion}

In this paper, we demonstrated that the modeling of ligaments is at least as important as the material parameters used for parenchyma model. It highlights the need for a good modeling of the boundary conditions. Thus, considering only the patient-specific data in simulations decreases their accuracy. This highlights the fact that the accuracy of the soft tissue parametrization has two key aspects: "patient-specific" as well as "scenario-specific". Moreover, we believe that in further studies and discussions, it would be interesting to focus on influence of boundary condition modeling in terms of their correct location as well as the model chosen to simulate their inherent mechanical behaviour. 


\section{References}

1. Simulation Open framework Architecture, http://www.sofa-framework.org

2. Bano, J., Hostettler, A., Nicolau, S., Cotin, S., Doignon, C., Wu, H.S., Huang, M.H., Soler, L., Marescaux, J.: Simulation of pneumoperitoneum for laparoscopic surgery planning. In: MICCAI (1). pp. 91-98 (2012)

3. Conte, C., Masson, C., Arnoux, P.J.: Inverse analysis and robustness evaluation for biological structure behaviour in FE simulation: application to the liver. Computer methods in biomechanics and biomedical engineering 15(9), 993-9 (Jan 2012)

4. Miller, K., amd Dane Lance, G.J., Wittek, A.: Total lagrangian explicit dynamics finite element algorithm for computing soft tissue deformation. Communications in Numerical Methods in Engineering 23(2), 121-134 (2007)

5. Miller, K., Lu, J.: On the prospect of patient-specific biomechanics without patientspecific properties of tissues. Journal of the mechanical behavior of biomedical materials 27, 154-66 (Nov 2013)

6. Payan, Y.: Soft tissue biomechanical modeling for computer assisted surgery, vol. 11. Springer (2012)

7. Peterlík, I., Duriez, C., Cotin, S.: Modeling and real-time simulation of a vascularized liver tissue. In: Proceedings of the 15th International Conference on Medical Image Computing and Computer-Assisted Intervention - Volume Part I. pp. 50-57. MICCAI'12, Springer-Verlag, Berlin, Heidelberg (2012)

8. Plantefeve, R., Peterlik, I., Courtecuisse, H., Trivisonne, R., Radoux, J.P., Cotin, S.: Atlas-based transfer of boundary conditions for biomechanical simulation. In: Proceedings of the 15th International Conference on Medical Image Computing and Computer-Assisted Intervention - Volume Part II. MICCAI'14 (2014)

9. Umale, S., Chatelin, S., Bourdet, N., Deck, C., Diana, M., Dhumane, P., Soler, L., Marescaux, J., R.Willinger: Experimental in vitro mechanical characterization of porcine glisson's capsule and hepatic veins. Journal of Biomechanics 44, 1678-1683 (2011) 